\title{
Predicting the Bond Strength of thin Steel Rebars in Concrete by Means of Artificial Neural Networks
}

\author{
Priscila F. S. Silva, Gray F. Moita, Eliene P. Carvalho \\ Mathematical and Computational Postgraduation Program, CEFET-MG \\ Av. Amazonas 7675, Belo Horizonte, MG, Brazil \\ 201422800040@aluno.cefetmg.br; gray@dppg.cefetmg.br; eliene@civil.cefetmg.br
}

\begin{abstract}
The bond strength between steel bars and concrete is one of the essential aspects of reinforced concrete structures and is generally affected by several factors. In this study, an experimental data set of 89 pull-out specimens was used to develop an artificial neural network (ANN). The data used in the modelling was arranged as 4 input parameters: bar surface, bar diameter $(\phi)$, concrete compressive strength $\left(f_{c}\right)$ and the anchorage length $\left(L_{d}\right)$. A comparative analysis was also conducted using the developed ANN model to establish the trend of the main influence variables on the bond capacity. The root mean squared error (RMSE) for the maximum applied load in the pull-out test, found on the testing data, was equal to $1.088 \mathrm{KN}$, and the R-squared was equal to 0.969 , for the proposed ANN model. Moreover, the study concluded that the proposed could be used to predicts the bond strength of thin bars.
\end{abstract}

Keywords: Steel-concrete bond; Thin rebars; Artificial neural network.

\section{Introduction}

Steel-concrete bonds are essential for the structural behaviour of reinforced concrete structures and an extensive range of parameters influences the characteristics of the steel-concrete interface [1]. Many authors have conducted experimental investigations into the most critical influence parameters upon the bond, and the bond characteristics are usually affected by the bar diameter, the anchorage length of bars, the concrete compressive strength, and the bar surface [2], [3]. As a phenomenon influenced by many variables, it is a challenge to establish how the steel-concrete adhesion can be described in standards used for reinforced concrete design [4].

Several scientific studies on this property have been performed since the 1940s [5], [6], among many other studies in this field. Generally, these studies refer to bars with diameters greater than $12.0 \mathrm{~mm}$ [7], [8]. However, few researches have evaluated the performance of reinforcing bars with diameters smaller than $10.0 \mathrm{~mm}$, which includes $5.0,6.3$, and $8.0 \mathrm{~mm}$ diameters, generally used in reinforced concrete elements [9].

One of the most used tests to evaluate the steel-concrete bond is the pull-out test, as described in RILEM-CEB RC [10]. The pull-out test consists of extracting a steel bar placed in the centre of a cubic concrete specimen, as shown in Fig. 1. The bond strength can be calculated with the pulling force measured at one end and the displacement is measured at the other end, as shown in Eq. (1):

$$
\tau=\frac{P}{\pi \cdot \phi \cdot L_{d}}
$$

where $\tau$ is the bond strength, $P$ is the applied load, $\phi$ is rebar diameter and $L_{d}$ is the anchorage length. 

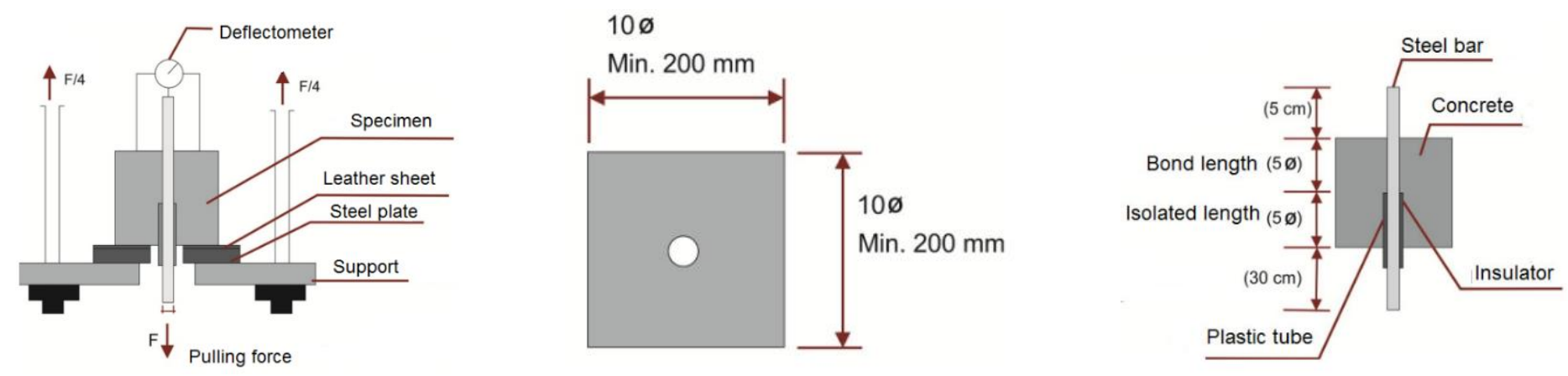

Fig. 1: Pull-out test set-up [10].

The size effect of rebars in the bond has been illustrated direct or indirectly by some researchers. Some of them discussed the theme of size effect on the steel-concrete bond, including bar diameter and the anchored length on the pull-out test. The small number of scientific studies on the bond of thin bars cast doubts on the parameters used to calculate the anchorage length of these bars in reinforced concrete elements.

Technological advancement usually allows engineering problems to be solved with machine learning, and its applications being good examples of fields explored with different expectations and realistic results. In general, artificial intelligence systems have shown their ability to solve real-life problems, particularly in nonlinear tasks [11].

Structural engineering has been a field of significant development through the implementation and testing of new computational models, predicting the different properties of concrete mixtures. In the case of behavioural models, pattern recognition is relevant and computational intelligence methods can be used. Bio-inspired models can also be an excellent aid to the design of structures for civil engineering [12]-[14]. The steel-concrete bond has also been an object of study using artificial intelligence in several works, but usually with rebars diameter greater than $10 \mathrm{~mm}$ [15]-[17]

This project focuses on the use of computational intelligence to analyse and develop a prediction model for the steel-concrete bond using an artificial neural network, emphasizing accuracy and efficiency, and the potential to deal with experimental data. This study aims to contribute to a new model to determine the bond strength, by establishing the maximum applied load, using artificial neural networks (ANNs).

\section{Artificial Neural Network}

ANNs are a typical example of a modern method that solves various engineering problems that cannot - or, at least, easily - be treated by traditional methods. The neural network can collect, memorise, analyse and process a large amount of data obtained through experimental tests [18], [19]

ANNs are one of the most useful computational models used in supervised regression tasks and learning classification. ANNs work primarily with three layers: the input layer, the hidden layers and the output layer. The performance of an ANN depends mostly on the performance of hidden layers.

The number of neurons in the input layer is a pattern usually presented to the neural network. Each neuron in the input layer must represent an independent variable that affects the outcome of the network. Therefore, the number of nodes in the input layer is equal to the number of inputs. The number of neurons in the output layer is directly related to the task that the neural network is performing.

\subsection{Activation functions}

When ANNs are built, it is vital to consider a suitable model architecture. In an ANN, neurons appear as

$$
z=w^{T} X+b
$$


followed by the activation function that determines whether the neuron is dispensed or follows to the output presented in the following equation:

$$
y=a(z)=a\left(w^{T} X+b\right)
$$

However, it is necessary to train the neural network to evaluate the results using some function error and to propagate through the neural network by updating weights (w) and bias (b). Therefore, derivatives of activation functions are used.

Understanding and choosing an appropriate activation function can minimize most significant problems. Other approaches that can be used are proper normalization, weight regularization, gradient clipping and the improvement of the architecture model.

In the following, a list of activation functions used in this project are presented:

- Softplus: The derivative of the softplus function is precisely the sigmoid function. The softplus function is

$$
f(x)=\ln \left(1+e^{x}\right)
$$

- Linear function (ELU): The linear function is presented in

$$
f(x)=x
$$

In this regression models, ELU should be used for the output layer and softplus for the other layers.

\subsection{Performance parameters:}

To evaluate the error obtained in each epoch, the mean square error

$$
M A E=\frac{1}{N} \sum_{i=1}^{N}\left|y_{i}-y\right|
$$

and the mean absolute error

$$
R M S E=\sqrt{M A E}=\sqrt{\frac{1}{N} \sum_{i=1}^{N}\left(y_{i}-y\right)^{2}}
$$

are commonly used, where $\hat{y}$ is the predicted value of $y$ and $y$ is the average value of $y$.

\section{Analysis and Results}

This work required the acquisition of reliable experimental data to determine the bond steel-concrete through computational intelligence. The database chosen was obtained from Carvalho et al [4]. This database presents 89 experimental tests. Four input parameters and one output parameter were used. The input parameters are as follows

- Concrete compressive strength $\left(f_{c}\right)$;

- Bar diameter $(\phi)$;

- Anchorage length $\left(L_{d}\right)$;

- Surface geometry of ribbed steel bars.

The output parameter is the maximum applied load $(\mathrm{kN})$.

The database used shows the maximums and minimums of input and output components, as presented in

Table 1. 
Table 1: Statistic parameters of data

\begin{tabular}{ccc}
\hline Model attributes & \multicolumn{2}{c}{ Values } \\
\cline { 2 - 3 } Compressive strength of concrete (MPa) & (Maximum) & (Minimum) \\
\hline Diameter $(\mathbf{m m})$ & 23 & 47 \\
\hline Anchorage length $(\mathbf{m m})$ & 6 & 10 \\
\hline Maximum applied load $(\mathbf{k N})$ & 30 & 100 \\
\hline
\end{tabular}

The type of surface geometry of steel bars present in this study is ribbed (type 1) and notched (type 2).

The presented data provided by Carvalho et al. [4] are consolidated and have a proper distribution for input and output variables. Still, it was necessary to implement the feature scaling technique to adequately standardize the data used.

A visualization of histograms and density boxes obtained from the database used is provided. This visualization aims to give a better idea of which method is more appropriate to obtain the result. Fig. 2 shows the histograms and density plots of the data used in the model. Fig. 3 presents the correlation matrix of the data used in the model.

The database visualization seeks to obtain a better understanding of the dataset to be studied. The intends to visualize correlations between inputs and outputs. Histograms can help us to form a better understanding of the data by showing information about each entry. The purpose is to estimate whether the database has a normal distribution or whether it is biased to the left or right. These figures help us to visualize and analyze the resources more effectively and facilitate choosing the most suitable computational models. Density plots are variables that provide an idea of each feature distribution in the dataset. With these plots, we can see a smooth distribution curve drawn over the top of each histogram. At last, box plots are an effective way to summarize the distribution of each available resource in the dataset. These boxes are useful because they give a better indication of the median value and the first and last quartile of the used data. 

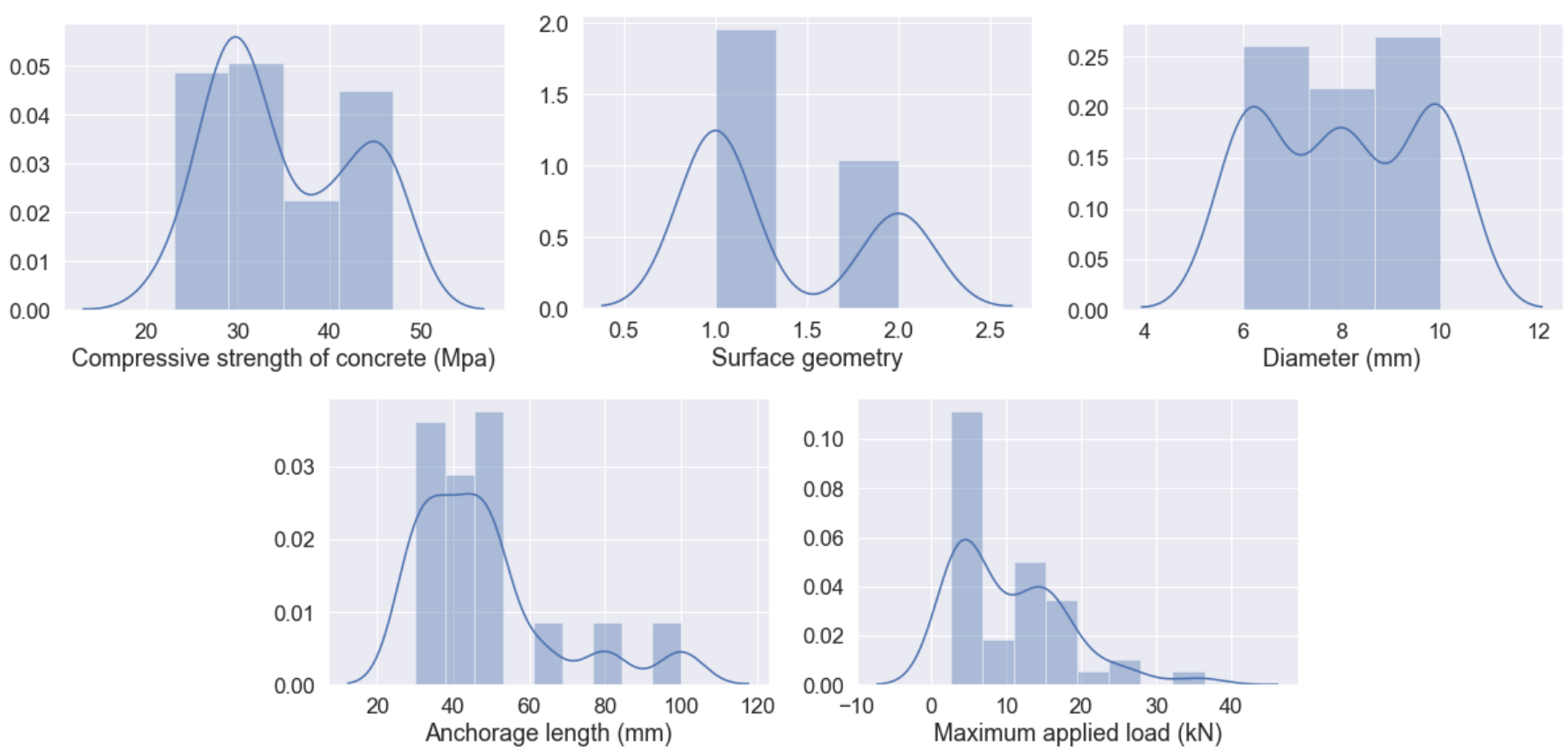

Fig. 2: Histograms and density plots.

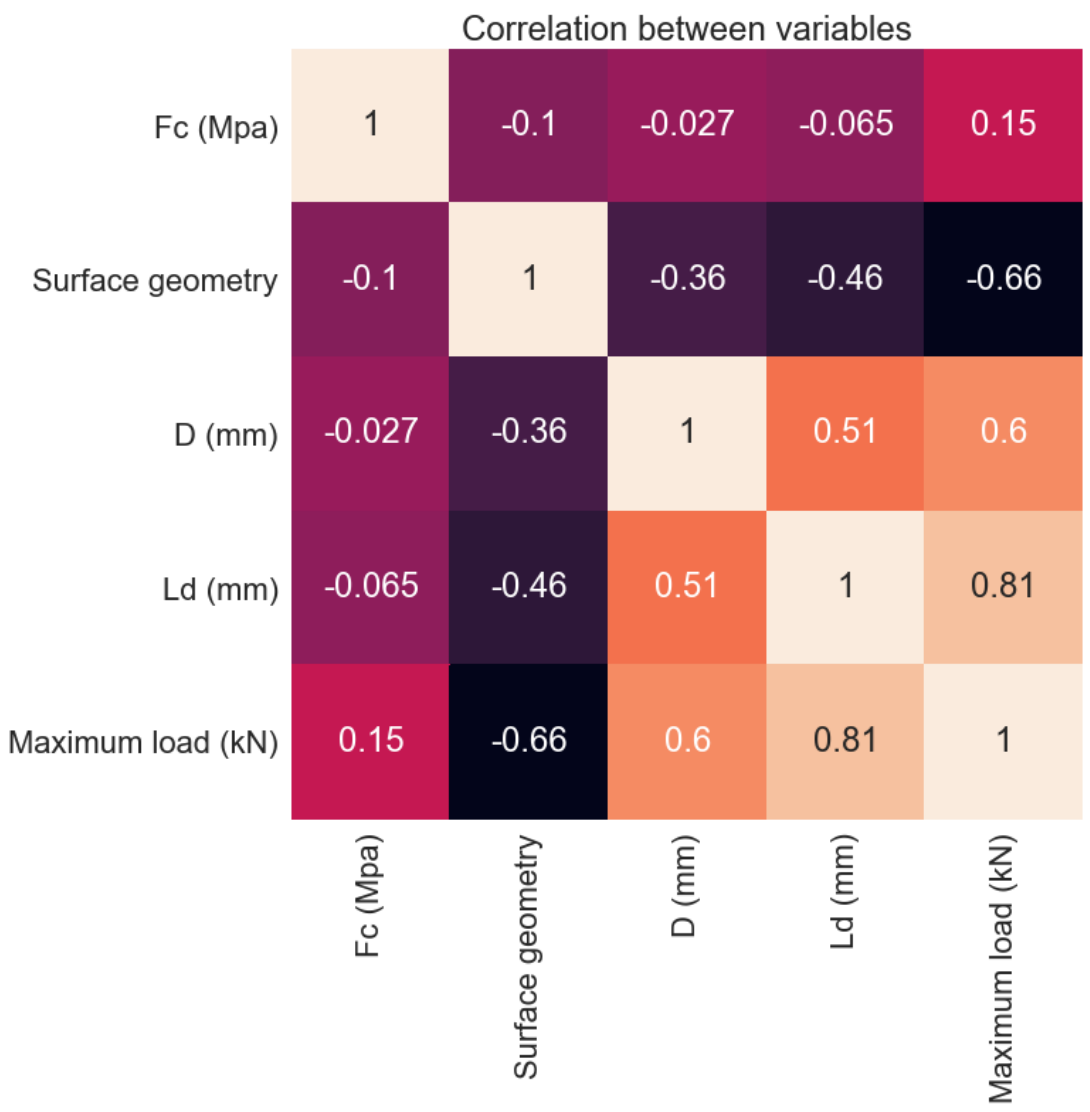

Fig. 3: Correlation matrix. 
The correlation matrix shows no correlation between the parameters bar surface and concrete compressive strength. It can be said that these components are mostly independent of each other. However, the anchorage length and the bar diameter correlate, as expected. All variable correlates with the maximum applied load, as also expected.

With the data analysis presented, the adequate architecture of the computational model was developed. The optimum architecture found has four neurons in the input layer. Furthermore, two intermediate layers, with ten neurons each, were used and one neuron was used in the output layer. The activation functions for the initial and intermediate layers were softplus. This decision was made based on its well-known performance for regression problems. Also, it was necessary to use a linear activation function in the last layer because the method is regression.

Fig. 4 shows the original and predicted values for the test data, and Fig. 5 shows the scatter of predicted and original values of bond strength test data for the proposed ANN model. The value found for $\mathrm{R}^{2}$ is equal to 0.969 and the RMSE is equal to 1.088. These figures reveal that the model used presents an excellent result with the given neural network.

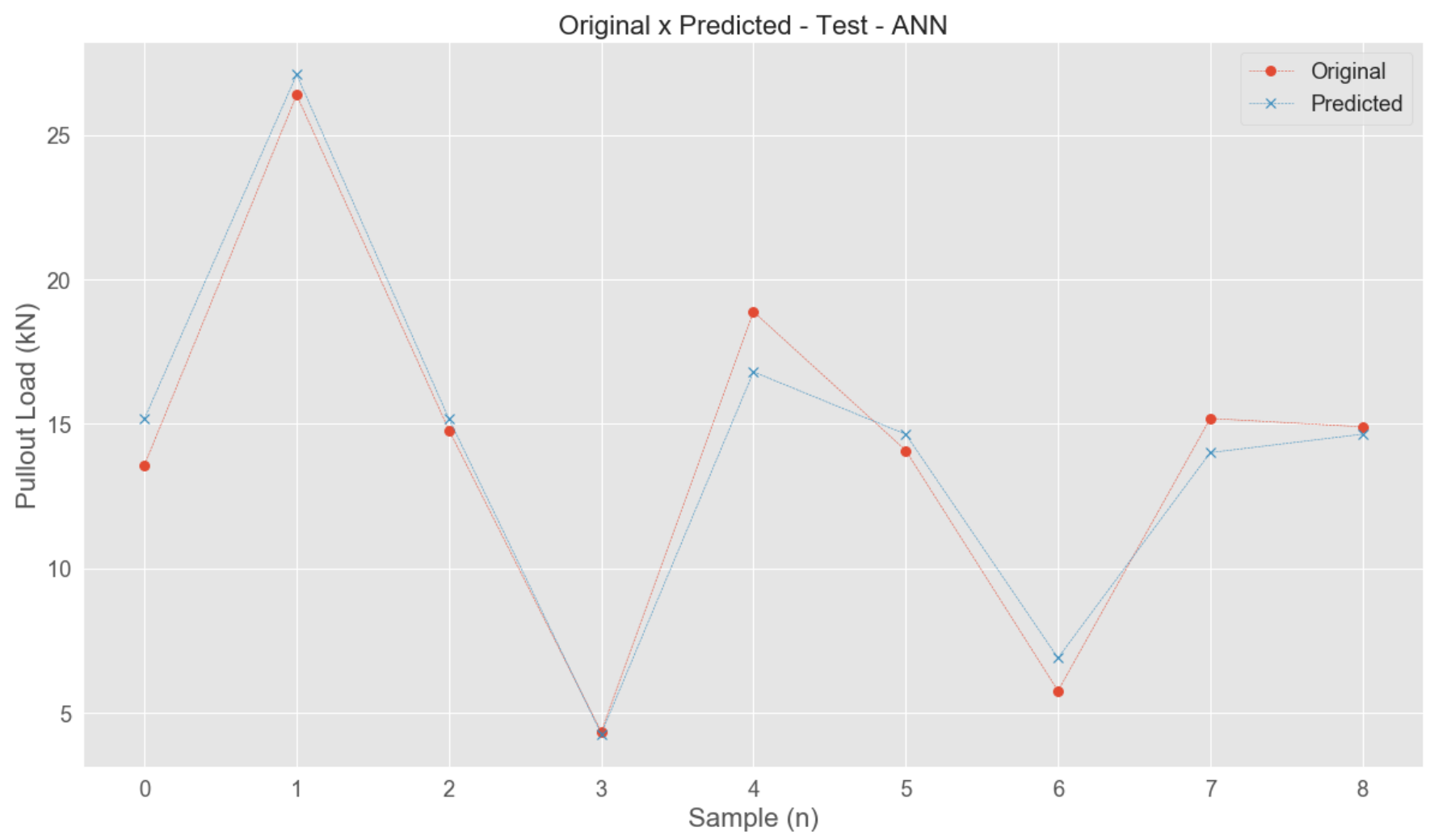

Fig. 4: Original versus expected results for ANN. 


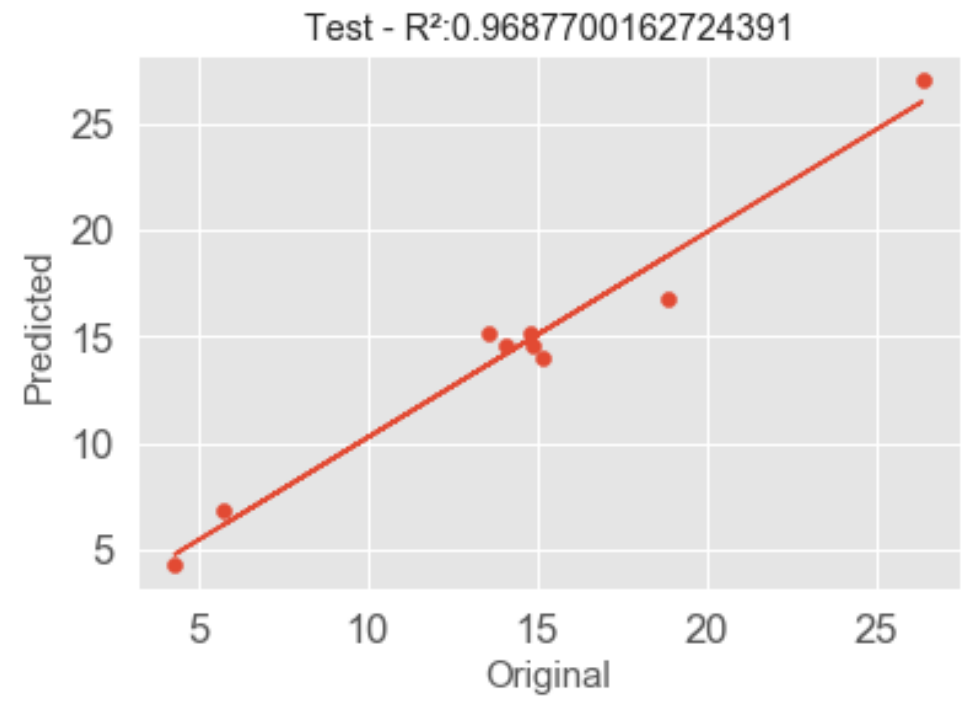

Fig. 5: Scatter of predicted and experimental values of bond strength.

The results obtained in this study are summarized in Table 2 .

Table 2: Obtained results for ANN.

\begin{tabular}{ccc}
\hline & $\begin{array}{c}\text { RMSE } \\
\text { (kN) }\end{array}$ & $\mathbf{R}^{\mathbf{2}}$ \\
\hline ANN & 1.088 & 0.969 \\
\hline
\end{tabular}

\section{Conclusions}

This work aimed to present the study of computational intelligence applied to define the bond strength from an original database obtained by Carvalho et al. [4]. A machine learning method, known as an artificial neural network, is used to find the maximum applied load. Data pre-processing and visualization methods were also used to improve the results.

The obtained results for the ANN show the best performance (RMSE $=1.088$ and $\mathrm{R}^{2}=0.969$ ). The computational intelligence model used is reliable to solve different complex problems, such as prediction problems. These models can be used to solve a specific problem when a deviation in available data is expected and accepted, and, also, when a defined methodology is not available. Therefore, to predict the properties of concrete, such as steel-concrete bond, with high reliability, conventional models can be replaced by computational intelligence models instead of using expensive experimental investigation.

Computational intelligence models can be used to predict the bond strength of concrete specimens, as shown in this study. The average errors found for the values predicted by the ANN and those predicted experimentally are highly consistent. Thus, the current study suggests an alternative approach to evaluate bond strength as opposed to destructive testing methods.

\section{Acknowledgments}

CAPES and CEFET-MG supported the work described in this paper.

\section{References}

[1] U. M. Angst et al., "The steel-concrete interface," Mater. Struct. Constr., vol. 50, no. 2, 2017, doi: 10.1617/s11527017-1010-1.

[2] C. A. Issa and O. Masri, "Numerical simulation of the bond behavior between concrete and steel reinforcing bars in 
specialty concrete," Int. J. Civil, Environ. Struct. Constr. Archit. Eng., vol. 9, no. 6, pp. 767-774, 2015.

[3] B. Liu, G. L. Bai, Z. H. Xu, J. F. Ma, and Y. Y. Han, "Experimental study and finite element modeling of bond behavior between recycled aggregate concrete and the shaped steel," Eng. Struct., vol. 201, no. September, p. 109840, 2019, doi: 10.1016/j.engstruct.2019.109840.

[4] E. P. Carvalho, E. G. Ferreira, J. C. da Cunha, C. de S. Rodrigues, and N. da S. Maia, "Experimental investigation of steel-concrete bond for thin reinforcing bars," Lat. Am. J. Solids Struct., vol. 14, no. 11, pp. 1932-1951, 2017, doi: 10.1590/1679-78254116.

[5] G. Rehm, The basic principles of the bond between steel and concrete. Cement and Concrete Association, 1968.

[6] R. M. Mains, "Measurement of the distribution of tensile and bond stresses along reinforcing bars," in Journal proceedings, 1951, vol. 48, no. 11, pp. 225-252.

[7] F. M. de Almeida Filho, S. De Nardin, and A. L. H. de C. El Debs, "Numerical approach of the steel-concrete bond behavior using pull-out models," Rev. Mater., vol. 24, no. 2, 2019, doi: 10.1590/s1517-707620190002.0656.

[8] F. M. De Almeida Filho, M. K. El Debs, and A. L. H. C. El Debs, "Bond-slip behavior of self-compacting concrete and vibrated concrete using pull-out and beam tests," Mater. Struct. Constr., vol. 41, no. 6, pp. 1073-1089, 2008, doi: 10.1617/s11527-007-9307-0.

[9] E. P. Carvalho, M. P. Miranda, D. S. G. Fernandes, and G. V. Alves, "Comparison of test methodologies to evaluate steel-concrete bond strength of thin reinforcing bar," Constr. Build. Mater., vol. 183, pp. 243-252, 2018, doi: 10.1016/j.conbuildmat.2018.06.109.

[10] R. CEB, "CEB/FIP Recommendations on reinforcement Steel for reinforced concrete," RC-6 Bond test Reinf. Steel 2. Pull-out test, Revis. Ed., 1983.

[11] N. D. Hoang, X. L. Tran, C. H. Le, and D. T. Nguyen, "A backpropagation artificial neural network software program for data classification in civil engineering developed in . NET framework," DTU J. Sci. Technol., vol. 03, no. 34, pp. 51-56, 2019.

[12] U. Reuter, A. Sultan, and D. S. Reischl, "A comparative study of machine learning approaches for modeling concrete failure surfaces," Adv. Eng. Softw., vol. 116, no. July 2017, pp. 67-79, 2018, doi: 10.1016/j.advengsoft.2017.11.006.

[13] P. G. Asteris, "Concrete compressive strength using artificial neural networks," Neural Comput. Appl., vol. 2, 2019, doi: 10.1007/s00521-019-04663-2.

[14] T. Nguyen-Sy, J. Wakim, Q. D. To, M. N. Vu, T. D. Nguyen, and T. T. Nguyen, "Predicting the compressive strength of concrete from its compositions and age using the extreme gradient boosting method," Constr. Build. Mater., vol. 260, p. 119757, 2020, doi: 10.1016/j.conbuildmat.2020.119757.

[15] Z. Dahou, Z. Mehdi Sbartaï, A. Castel, and F. Ghomari, "Artificial neural network model for steel-concrete bond prediction," Eng. Struct., vol. 31, no. 8, pp. 1724-1733, 2009, doi: 10.1016/j.engstruct.2009.02.010.

[16] E. M. Golafshani, A. Rahai, and M. H. Sebt, "Artificial neural network and genetic programming for predicting the bond strength of GFRP bars in concrete," Mater. Struct. Constr., vol. 48, no. 5, pp. 1581-1602, 2015, doi: 10.1617/s11527-014-0256-0.

[17] M. Makni, A. Daoud, M. A. Karray, and M. Lorrain, "Artificial neural network for the prediction of the steel-concrete bond behaviour," Eur. J. Environ. Civ. Eng., vol. 18, no. 8, pp. 862-881, 2014, doi: 10.1080/19648189.2014.909745.

[18] J. J. Hopfield, "Artificial neural networks," IEEE Circuits Devices Mag., vol. 4, no. 5, pp. 3-10, 1988.

[19] W. S. Sarle, "Neural networks and statistical models," Proc. Ninet. Annu. SAS Users Gr. Int. Conf., 1994. 